causes patients to die, but also increases the work load of doctors, affects the sickbed-turnover in the hospital, and increases the cost of care and the economic burden on patients.

From January 1997 to October 1998, 452 patients were discharged from the tumor faculty of Wuhan No. 4 Hospital; 75 (17\%) had NI, as compared to $5 \%$ of nontumor patients. To estimate the effect of these NIs on costs, we compared 22 lung cancer patients with NI to 22 lung cancer patients without NI (Table).

It can be seen that there are remarkable differences between the two groups in costs, particularly for medicine and transfusion.

It should be pointed out that NIs also give rise to a great deal of indirect economic loss; for example, sufferers create less wealth for the country because they are absent or dead, and their relations visit, consuming resources. Therefore the actual loss is larger than this.

Controlling NI calls for prevention and countermeasures. First, we must increase the patients' own resistance. We give them a great deal of sustained treatment using combined Chinese and Western medicines. Second, we must use antibiotics with reason. Third, we must reduce invasive operations and treatment. Most importantly, we must build the perfect system of family sickbed service, so that doctors and nurses can cure them in their family. It not only saves a great number of costs but also avoids cross-infection. It fits our country's situation completely.

When I see the bad patient who emerge their life in their eyes, I really want to say: "We hope the life tree will always be green!"

Lin Cao, MBBS Wuhan No. 4 Hospital Wuhan, Hubei, China

\section{Surgical-Site \\ Complications Associated With a Morphine Nerve Paste Used for Postoperative Pain Control After Laminectomy}

To the Editor:

It was with interest that I read the

TABLE

Costs (IN RMB) of Treatment for Lung Cancer Patients With and Without NOSOCOMIAL INFECTTON (NI)

\begin{tabular}{lrrr}
\hline Item Costs & \multicolumn{1}{c}{ NI } & No NI & Difference \\
\hline Patients & 22 & 22 & \\
Medicine & 230,680 & 208,602 & 22,078 \\
Transfusion & 2,226 & 580 & 1,646 \\
Inspection & 22,850 & 18,000 & 4,850 \\
Radiology & 2,558 & 2,210 & 348 \\
Surgery & 98,214 & 59,820 & 38,394 \\
Oxygen therapy & 5,028 & 8,936 & $-3,908$ \\
Bed-stay costs & 36,106 & 26,586 & 9,518 \\
Hospital-days & 2,132 & 1,494 & 638
\end{tabular}

Abbreviations: NI, nosocomial infection; RMB, the unit of currency in China.

article by Kramer and colleagues ${ }^{1}$ documenting their disappointment with morphine nerve paste and their suspicion for delayed wound healing with increased postoperative morbidity. We recently published the results of a prospective, double-blind, randomized trial evaluating a similar paste in patients undergoing lumbar decompressive surgery. ${ }^{2}$ Our experience with the paste was much more positive. While three patients in the actively treated group had minor wound complications treated locally, none required debridement or re-exploration. The decrease in both inpatient and outpatient postoperative narcotic analgesic consumption was statistically significant for up to 6 weeks after surgery. In addition, McGill pain scores and the SF-36 General Health Perception questionnaire also were significantly better in the treated group to 6 weeks.

In an ongoing prospective, doubleblind, follow-up study at the University of Calgary, over 100 patients have been randomized to active or placebo groups. We have experienced only 1 patient with a wound complication in this entire cohort and remain blinded to that patient's treatment status. These results echo those of the independent study initially reported by Needham. ${ }^{3}$ Kramer and colleagues report an "epidemic" of wound complications; we certainly agree with their use of this term. However, their experience is not reproduced at any of three independent institutions ( $\mathrm{RJH}$, unpublished data, 1999). ${ }^{2,3}$ Hence, the epidemic described by Kramer et al is more likely related to conditions specific to "hospital A" or differences in application technique.
We maintain, based on results of prospective, controlled, randomized trials with follow-up of up to 1 year, that the morphine paste as described by Needham can be used both safely and effectively. Proper watertight closure of the lumbodorsal fascia and irrigation of the subcutaneous compartment to remove residual paste compound are critical to proper application., ${ }^{2,3}$ These steps are felt to be very important in reducing the potential for postoperative third spacing of extracellular fluid, possibly encouraged by the hyperosmolar properties of the paste.

\section{REFERENCES}

1. Kramer $\mathrm{MH}$, Mangram AJ, Pearson $\mathrm{ML}$, Jarvis WR. Surgical-site complications with a morphine nerve paste used for postoperative pain control after laminectomy. Infect Control Hosp Epidemiol 1999;20:183-186.

2. Hurlbert RJ, Theodore N, Drabier JB, Magwood AM, Sonntag VK A prospective randomized double-blind controlled trial to evaluate the efficacy of an analgesic epidural paste following lumbar decompressive surgery. J Neurosurg 1999;90(4 suppl):191-197.

3. Needham CW. Painless lumbar surgery: morphine nerve paste. Conn Med 1996; 60:141-143.

R. John Hurlbert, MD, PhD

University of Calgary Spine Program Calgary, Alberta, Canada

\section{To the Editor:}

Dr. Hurlbert was kind enough to provide me with a copy of his letter to you. I agree with Dr. Hurlbert. 1,2

Approximately $1 \frac{1}{2}$ to 2 years ago, $I$ received a call from an orthopedic surgeon who was having some wound healing problems after employing morphine nerve paste. I asked him if he was following the instructions set forth 
in my article ${ }^{1}$ about personally and closely observing the nurses during mixing of the paste, then immediately applying the paste. It was apparent to me at the time of our conversation that these items were not being given his careful attention. I told the orthopedic surgeon that he was ignoring my method. He would not know if the right amounts of the drugs involved were mixed or if they might have been contaminated in the mixing process. My technique is not merely a recipe but a method. The epidural paste must be mixed under the surgeon's eye and immediately applied. The field should be dry and the dura intact. The lumbar fascia should be closed in a watertight way. This ensures that the liquid reservoir of morphine stays in the subfascial space and keeps the epidural paste away from the fascia and skin. It has long been known that Avitene molecules should be kept away from skin.

My clear impression after talking with the orthopedic surgeon was that my detailed method was not being followed. Although "hospital A" was never identified in Kramer's article, ${ }^{3}$ it is my belief it is the same orthopedic hospital where my paste was misused. In the article, Kramer et al indicated that sometimes the paste was premixed (not under the eye of the surgeon) and applied later (in one case as much as 45 minutes later). A neurosurgeon putting a foreign body (eg, a shunt) into the human body does not leave it open, exposed to the air, etc. He takes the shunt out of its sterile package, fills it with fluid to test it, then immediately puts it into the body.

I devised the paste 3 years ago, to be used in lumbar laminectomy for ruptured discs and stenosis cases, not for large orthopedic instrumented cases where the orthopedist will use metal, screws, or cages, where the surgery lasts many hours, where blood loss and blood transfusions are common, and where drains are employed. A bloody field, the use of drains, and many hours, as well as foreign bodies, increase the risk of infection. The use of drains because of bleeding also removes the liquid morphine reservoir that separates the closed lumbar fascia from the epidural paste. The use of drains therefore shortens the effectiveness of the nerve paste, which itself contains only $1 \mu \mathrm{g}$ of morphine. As Dr. Hurlbert points out, "hospital A" may have problems unique to it, related to sterilization techniques, staffing, etc.

When the method I described in my paper in 1996 is carefully followed, as it was in the double-blind study at the Barrow Neurological Institute in Phoenix, Arizona, the results are generally excellent. No increase in wound problems should be anticipated, and postoperative pains are usually eliminated. This was shown in the doubleblind, controlled Barrow study.

A recipe is not just a list of ingredients. A specific method for safe application of the paste was set forth. When the method is not followed with care then problems may occur.

\section{REFERENCES}

1. Needham $\mathrm{CW}$. Painless lumbar surgery: morphine nerve paste. Conn Med 1996;60: 141-143.

2. Hurlbert RJ, Theodore N, Drabier JB, Magwood AM, Sonntag VK A prospective randomized double-blind controlled trial to evaluate the efficacy of an analgesic epidural paste following lumbar decompressive surgery. J Neurosurg 1999;90(4 suppl):191-197.

3. Kramer MH, Mangram AJ, Pearson ML, Jarvis WR. Surgical-site complications with a morphine nerve paste used for postoperative pain control after laminectomy. Infect Control Hosp Epidemiol 1999;20:183-186.

Charles W. Needham, MD Norwalk, Connecticut

\section{The authors reply.}

Hurlbert and colleagues have published the results of a randomized placebo-controlled trial in which they assessed the efficacy of morphine nerve paste for postoperative analgesia after decompressive lumbar laminectomy. ${ }^{1}$ Their findings show that morphine nerve paste significantly improves postoperative pain control and reduces overall analgesic consumption. However, we believe that their study, like our investigation, raises some important issues about the safety of this new analgesic preparation.

In their trial, Hurlbert et al studied a total of 60 patients: 30 received paste and 30 received placebo. Of 30 patients who received paste, 1 developed serious drainage, and 2 had superficial surgical-site infections, complications similar to those experienced by patients in our report. No such complications were observed among the control group. Furthermore, the rate of surgical-site complications among the patients who received the paste in Hurlbert's study (10\%) was similar to the rate detected among the paste recipients in our investigation (11.5\%). ${ }^{2}$ We found that the rate of complications was significantly higher in patients who received the paste than in those who did not $(11.5 \%$ vs $1.5 \%$, $P<.001)$. In Hurlbert's study, the difference in the rate of surgical-site complications between the treatment (10\%) and nontreatment (0\%) groups did not reach statistical significance, suggesting that the number of patients studied in their trial may have been adequate to assess the efficacy of the paste, but not necessarily adverse events of low frequency.

We also believe that Hurlbert's report highlights the variability in procedural practices that may occur when the paste is used. First, neither Hurlbert's trial nor the original case series describing the paste ${ }^{3}$ specified the amount of paste used on a single patient. Similarly, we could not document the amount of paste used during procedures in our investigation. Second, Hurlbert et al report the need for thorough irrigation of the subcutaneous tissues after closure of the lumbodorsal fascia to prevent sterile fluid accumulation, a recommendation not made in Needham's original publication regarding the paste. Lastly, all of the procedures in Hurlbert's trial were done by a single surgeon, and the paste was always applied by the same person. By contrast, in our investigation, multiple surgeons performed the procedures in which the paste was used. We agree that variability in procedural practices likely influences the risk of surgical-site complications when the paste is used and that the surgical-site complications described in our article may be attributable to institution-specific conditions. Nevertheless, we believe that the circumstances at this hospital more likely represent the variability in surgical technique and other surgical practices that exist within and between institutions than do the circumstances described in Hurlbert's controlled trial.

Morphine nerve paste appears to be an innovative and efficacious approach to postoperative pain control after laminectomy. However, conclusions about the safety of the product can not be appropriately made until the use of the paste becomes more widespread or larger multicenter studies are done. Our report was intended to alert the medical community about the possibility of surgical-site complications when morphine nerve paste is used. Our findings and those reported by Hurlbert et al are an important contribution to the scientific discussion about the potential benefits and risks 